\title{
ON THE $L^{1}$ NORM AND THE MEAN VALUE OF A TRIGONOMETRIC SERIES
}

\author{
L. C. KURTZ AND S. M. SHAH
}

1. Introduction. In a recent paper [6] S. Uchiyama has derived lower bounds for

$$
\int_{0}^{1}\left|\sum_{k=1}^{n} \phi_{k}(t) c_{k} e\left(m_{k} x\right)\right| d x
$$

where $\phi_{k}$ is the $k$ th Rademacher function, $\left\{m_{k}\right\}$ is a sequence of distinct integers, and $e\left(m_{k} x\right)=\exp \left(2 \pi i m_{k} x\right)$. Uchiyama's results hold except for values of $t$ in sets of arbitrarily small measure; these exceptional sets may include the values of $t$, near the origin, for which $\phi_{k}(t)=1$. We find here lower bounds for

$$
\int_{0}^{1}\left|\sum_{k=1}^{n} c_{k} e\left(m_{k} x\right)\right| d x
$$

which correspond to Uchiyama's results, but for these values of $t$. We have to put some conditions on the sequence $\left\{m_{k}\right\}$; simple examples show that our bounds cannot hold in general.

S. Chowla [1] conjectured that, for any sequence $\left\{m_{k}\right\}$ of increasing positive integers

$$
\min _{0 \leqq x<1} \sum_{k=1}^{n} \cos 2 \pi m_{k} x<-c n^{1 / 2}
$$

for some absolute constant $c>0$. S. Uchiyama [6] proved that given $n$ distinct integers $m_{1}, \cdots, m_{n}$, there exists always a subset $m_{j_{1}}$, $\cdots, m_{j_{r}}$ of $m_{1}, \cdots, m_{n}$, for which

$$
\min _{0 \leqq x<1} \sum_{i=1}^{r} \cos 2 \pi m_{j_{i}} x<-\frac{1}{4}\left(\frac{1}{6}\right)^{1 / 2} n^{1 / 2}=(-0.102 \cdots) n^{1 / 2} .
$$

We prove here that if $\left\{m_{k}\right\}$ is an admissible sequence (for definition, see below), then

$$
\min _{0 \leqq x<1} \sum_{k=1}^{n} \cos 2 \pi m_{k} x<-\frac{1}{8} n^{1 / 2}=(-0.125) n^{1 / 2} .
$$

Presented to the Society, September 1, 1967 under the title $O n$ the $L^{1}$ norm and the mean value of a trigonometric polynomial; received by the editors June 10, 1967. 
2. Admissible sequences. We will say that a sequence $\left\{m_{k}\right\}$ of positive integers is admissible provided that $\left\{m_{k}\right\}$ is strictly increasing and $m_{k}-m_{j}+m_{l}-m_{p} \neq 0$ if $k \neq j, k \neq p$ and $j \neq l$ all hold. Note that this condition automatically holds if $l=p$ since $\left\{m_{k}\right\}$ is strictly increasing. Hence we shall assume $l \neq p$. Similarly if $k=l$ the condition is satisfied when $j=p$. There are many sequences which are admissible, as the following lemma shows.

Leмma. If $\left\{m_{k}\right\}$ is a sequence of positive integers such that $m_{k+1} \mid m_{k}$ $\geqq 2$, then $\left\{m_{k}\right\}$ is admissible.

Proof. Let $m_{k}=\max \left(m_{k}, m_{j}, m_{p}, m_{l}\right)$. Then

$$
m_{k}-m_{j}+m_{l}-m_{p}>m_{k}-m_{j}-m_{p} \geqq m_{k}-2 \max \left(m_{j}, m_{p}\right) \geqq 0 .
$$

If $m_{j}=\max \left(m_{k}, m_{j}, m_{p}, m_{l}\right)$, then the same argument shows that $m_{k}-m_{j}+m_{l}-m_{p} \neq 0$.

Remarks. For some deep results of Erdös, Chowla and others for similarly defined $B_{h}$-sequences $(h \geqq 2)$, see [3, pp. 76-97].

3. Theorems. We let $S_{n}(x)=\sum_{k=1}^{n} c_{k} e\left(m_{k} x\right), R_{n}=\sum_{k=1}^{n}\left|c_{k}\right|^{2}$ and $T_{n}=\sum_{k=1}^{n}\left|c_{k}\right|^{4}$.

THEOREM 1. If $\left\{m_{k}\right\}$ is an admissible sequence, then

$$
\int_{0}^{1}\left|S_{n}(x)\right| d x \geqq\left(\frac{R_{n}}{2-1 / n}\right)^{1 / 2}, \quad n=1,2, \cdots .
$$

Proof. It is easy to see that

$$
\int_{0}^{1}\left|S_{n}(x)\right|^{2} d x=R_{n}
$$

Also,

$$
\begin{aligned}
& \int_{0}^{1}\left|S_{n}(x)\right|^{4} d x=\int_{0}^{1}\left\{\sum_{k, j=1}^{n} c_{k} \bar{c}_{j} e\left(\left(m_{k}-m_{j}\right) x\right)\right\}^{2} d x \\
& =\int_{0}^{1} \sum_{k, j=1}^{n}\left\{\sum_{l, p=1}^{n} c_{k} \bar{c}_{j} c_{l} \bar{c}_{p} e\left(\left(m_{k}-m_{j}+m_{l}-m_{p}\right) x\right)\right\} d x .
\end{aligned}
$$

We break this sum into three parts:

(A) The terms with $k=j$ give

$$
\int_{0}^{1} \sum_{k=1}^{n}\left\{\sum_{l, p=1}^{n}\left|c_{k}\right|^{2} c_{l} \bar{c}_{p} e\left(m_{l}-m_{p}\right) x\right\} d x=\sum_{k=1}^{n} \sum_{l=1}^{n}\left|c_{k}\right|^{2}\left|c_{l}\right|^{2}
$$

(B) When $k \neq j$ we have some terms when $k=p$ and $j=l$. These terms give the sum $\sum_{k, j=1 ; k \neq j}\left|c_{k}\right|^{2}\left|c_{j}\right|^{2}$. 
(C) The remaining terms are given by

$$
\int_{0}^{1} \sum_{k, j=1 ; k \neq j}^{n}\left\{\sum_{l, p=1}^{n} c_{k} \bar{c}_{j} c_{l} \bar{c}_{p} e\left(\left(m_{k}-m_{j}+m_{l}-m_{p}\right) x\right)\right\} d x
$$

where either $k \neq p$ or $j \neq l$. In fact we may take $k \neq p$ and $j \neq l$ for if $k \neq p$ and $j=l$, the corresponding integral vanishes. Thus we have $k \neq j, k \neq p, j \neq l$ in (C); so $m_{k}-m_{j}+m_{l}-m_{p} \neq 0$ since $\left\{m_{k}\right\}$ is admissible, and the integral vanishes. This shows that

$$
\int_{0}^{1}\left|S_{n}(x)\right|^{4} d x=\sum_{k, l=1}^{n}\left|c_{k}\right|^{2}\left|c_{l}\right|^{2}+\sum_{k, l=1 ; k \neq l}^{n}\left|c_{k}\right|^{2}\left|c_{l}\right|^{2}=2 R_{n}^{2}-T_{n} .
$$

Hölder's inequality now yields the result,

$$
\begin{aligned}
\left(\int_{0}^{1}\left|S_{n}(x)\right| d x\right)^{2 / 3} & \geqq \int_{0}^{1}\left|S_{n}(x)\right|^{2} d x /\left(\int_{0}^{1}\left|S_{n}(x)\right|^{4} d x\right)^{1 / 3} \\
& =R_{n} /\left(2 R_{n}{ }^{2}-T_{n}\right)^{1 / 3}
\end{aligned}
$$

Hence

$$
\int_{0}^{1}\left|S_{n}(x)\right| d x \geqq R_{n}^{3 / 2} /\left(2 R_{n}^{2}-T_{n}\right)^{1 / 2} \geqq R_{n}^{1 / 2} /(2-1 / n)^{1 / 2} .
$$

Note that there is an equality sign when $n=1$. Also we have

$$
(2-1 / n)^{-1 / 2} \leqq\left\|S_{n}\right\|_{1} /\left\|S_{n}\right\|_{2} \leqq 1,
$$

where $\left\|S_{n}\right\|_{p}(p=1,2)$ denotes $L^{p}$ norm of $S_{n}$.

We can get a similar result for real series

(3) $\quad T_{n}(x, \alpha)=T_{n}(x)=\sum_{k=1}^{n} \rho_{k} \cos 2 \pi\left(m_{k} x+\alpha_{k}\right), \quad \rho_{k} \geqq 0, \quad \alpha_{k}$ real.

ThEOREM 2. If $\left\{m_{k}\right\}$ is an admissible sequence, then

$$
\begin{aligned}
\int_{0}^{1}\left|T_{n}(x)\right| d x \geqq & \left(\sum_{1}^{n} \rho_{k}^{2}\right)^{1 / 2} \\
& \cdot \frac{1}{2^{3 / 2}(2-1 / n)^{1 / 2}}, \quad n=1,2, \cdots .
\end{aligned}
$$

Proof. We have

$$
\int_{0}^{1}\left|T_{n}(x)\right|^{2} d x=\frac{1}{2} \sum_{k=1}^{n} \rho_{k}^{2} .
$$

If $U_{n}(x)=\sum_{k=1}^{n}\left\{\rho_{k} e\left(m_{k} x+\alpha_{k}\right)\right\}$, then $T_{n}(x)=\operatorname{Re} U_{n}(x)$. By Theorem 1 , 
1026

$$
\begin{aligned}
\int_{0}^{1}\left|U_{n}(x)\right|^{4} d x & =2\left(\sum_{k=1}^{n}\left|\rho_{k} e\left(\alpha_{k}\right)\right|^{2}\right)^{2}-\sum_{k=1}^{n}\left|\rho_{k} e\left(\alpha_{k}\right)\right|^{4} \\
& =2\left(\sum_{k=1}^{n} \rho_{k}^{2}\right)^{2}-\sum_{1}^{n} \rho_{k}^{4}
\end{aligned}
$$

But $\left|T_{n}(x)\right| \leqq\left|U_{n}(x)\right|$ and Hölder's inequality now gives

$$
\begin{aligned}
& \left(\int_{0}^{1}\left|T_{n}(x)\right| d x\right)^{2 / 3} \\
& \geqq \frac{\int_{0}^{1}\left|T_{n}(x)\right|^{2} d x}{\left(\int_{0}^{1}\left|T_{n}(x)\right|^{4} d x\right)^{1 / 3}} \geqq \frac{\int_{0}^{1}\left|T_{n}(x)\right|^{2} d x}{\left(\int_{0}^{1}\left|U_{n}(x)\right|^{4} d x\right)^{1 / 3}} \\
& \geqq \frac{\frac{1}{2} \sum_{k=1}^{n} \rho_{k}^{2}}{\left[(2-1 / n)^{1 / 3}\left(\sum_{1}^{n} \rho_{k}^{2}\right)^{2 / 3}\right]}=\left(\sum_{k=1}^{n} \rho_{k}^{2}\right)^{1 / 3} \frac{1}{2} \frac{1}{(2-1 / n)^{1 / 3}},
\end{aligned}
$$

and ${ }^{7}(4)$ is proved.

ThEOREM 3. If $\left\{m_{k}\right\}$ is an admissible sequence, then

$$
\min _{0 \leq x<1} T_{n}(x) \leqq-\frac{1}{2^{5 / 2}(2-1 / n)^{1 / 2}}\left(\sum_{1}^{n} \rho_{k}^{2}\right)^{1 / 2} .
$$

Proof. Write

$$
T_{n}^{+}=\max \left(T_{n}, 0\right), \quad T_{n}^{-}=-\min \left(T_{n}, 0\right) .
$$

Then

$$
\begin{aligned}
\int_{0}^{1}\left|T_{n}\right| d x & =\int_{0}^{1} T_{n}^{+} d x+\int_{0}^{1} T_{n}^{-} d x \\
\int_{0}^{1} T_{n} d x & =\int_{0}^{1} T_{n}^{+} d x-\int_{0}^{1} T_{n}^{-} d x=0 .
\end{aligned}
$$

Hence

$$
2 \int_{0}^{1} T_{n}^{-} d x=\int_{0}^{1}\left|T_{n}(x)\right| d x .
$$

But $T_{n}^{-}(x) \leqq-\min _{0 \leqq x \leqq 1} T_{n}(x)$. Hence 


$$
\frac{1}{2^{5 / 2}(2-1 / n)^{1 / 2}}\left(\sum_{1}^{n} \rho_{k}^{2}\right)^{1 / 2} \leqq \int_{0}^{1} T_{n}^{-}(x) d x \leqq-\min _{0 \leq x \leq 1} T_{n}(x),
$$

and the theorem is proved.

COROLlaRy. If $\left\{m_{k}\right\}$ is an admissible sequence, then

(6) $\min _{0 \leq x<1} \sum_{1}^{n} \cos 2 \pi m_{k} x \leqq-2^{-5 / 2}\left(2-\frac{1}{n}\right)^{-1 / 2} n^{1 / 2}<-\frac{1}{8} n^{1 / 2}$.

We now consider this minimum when $\left\{m_{k}\right\}$ is not necessarily an admissible sequence. Let $\beta_{0}$ be the unique root of the equation

$$
I(x)=\int_{0}^{3 \pi / 2} \frac{\cos u}{u^{x}} d u=0 .
$$

The value of $\beta_{0}(=.30844 \cdots)$ has been calculated (cf. [2], [4]) to fifteen places of decimal.

TheOREM 4. Write

$$
m(n)=\min _{0 \leq x<1} T_{n}(x, 0)=\min _{0 \leq x<1} \sum_{k=1}^{n} \rho_{k} \cos 2 \pi m_{k} x,
$$

and let $0<\beta<\beta_{0}, 1 \leqq b<1 /(1-\beta), 0<\gamma<1-b(1-\beta)$. Suppose that

(8) $1 \leqq m_{1}<m_{2}<\cdots, \quad m_{k}<K k^{b} \rho_{k}^{1 /(1-\beta)}, \quad k=1,2, \cdots$, where $K$ is a constant. Then

$$
\limsup _{n \rightarrow \infty}\left\{-m(n) / n^{\gamma}\right\}>0 .
$$

We omit the proof which is similar to that of Theorem 3 of [2].

REMARKS. A result similar to Theorem 4 can be proved for

$$
\min _{0 \leq x<1} \sum_{1}^{n} \rho_{k} \cos \left(2 \pi m_{k} x+2 \pi \alpha_{k}\right),
$$

provided we put a suitable condition on $\alpha_{k}$ (cf. [2], [5]).

ExAmPLE. Let $1 \leqq m_{1}<m_{2}<\cdots, m_{k}=O\left(k^{1+\epsilon}\right), \rho_{k}>0, k=1,2, \cdots$, $\rho_{k}{ }^{-1}=O\left(k^{\epsilon}\right)$ for every $\epsilon>0$. Then the condition (8) is satisfied with $b>1$. Hence (9) holds with any number $\gamma<\beta_{0}$. This extends a result of Chowla [1, p. 131].

\section{REFERENCES}

1. S. Chowla, Some applications of a method of A. Selberg, J. Reine Angew. Math. 217 (1965), 128-132. 
2. J. Chidambaraswamy and S. M. Shah, Trigonometric series with nonnegative partial sums, J. Reine Angew. Math. 229 (1968), 163-169.

3. H. Halberstam and K. F. Roth, Sequences, Vol. I, Clarendon Press, Oxford, 1966.

4. R. D. Halbgewachs and S. M. Shah, Trigonometric sums and Fresnel-type integrals, Proc. Indian Acad. Sci. Sect. A 65 (1967), 227-232.

5. S. M. Shah, Trigonometric series with non-negative partial sums, Proc. Sympos. Pure Math., Vol. 11, Amer. Math. Soc., Providence, R. I., 1968.

6. S. Uchiyama, On the mean modulus of trigonometric polynomials whose coefficients have random signs, Proc. Amer. Math. Soc. 16 (1965), 1185-1190.

UNIVERSITY OF KENTUCKY 